\title{
Influence of dose on particle size of colloidal silver nanoparticles synthesized by gamma radiation.
}

\begin{abstract}
Colloidal silver nanoparticles were synthesized by $\gamma$-irradiation-induced reduction method of an aqueous solution containing silver nitrate as a precursor in various concentrations between $7.40 \times 10-4$ and $1.84 \times 10-3 \mathrm{M}$, polyvinyl pyrrolidone for capping colloidal nanoparticles, isopropanol as radical scavenger of hydroxyl radicals and deionised water as a solvent. The irradiations were carried out in a 60Co $\gamma$ source chamber at doses up to $70 \mathrm{kGy}$. The optical absorption spectra were measured using UV-vis spectrophotometer and used to study the particle distribution and electronic structure of silver nanoparticles. As the radiation dose increases from 10 to $70 \mathrm{kGy}$, the absorption intensity increases with increasing dose. The absorption peak $\lambda \max$ blue shifted from 410 to $403 \mathrm{~nm}$ correspond to the increase of absorption conduction electron energy from 3.02 to $3.08 \mathrm{eV}$, indicating the particle size decreases with increasing dose. The particle size was determined by photon cross correlation spectroscopy and the results showed that the particle diameter decreases exponentially with the increase of dose. The transmission electron microscopy images were taken at doses of 20 and $60 \mathrm{kGy}$ and the results confirmed that as the dose increases the diameter of colloidal silver nanoparticle decreases and the particle distribution increases.
\end{abstract}

Keyword: Silver nanoparticles; $\gamma$-Irradiation; Particle size; Particle distribution. 